\title{
Vibrotactile Jacket for Perception Enhancement
}

\author{
Sylvain Cardin, Daniel Thalmann \\ VRLab , Ecole Polytechnique Fédérale de Lausanne \\ 1015 Lausanne - Switzerland \\ sylvain.cardin@epfl.ch \\ daniel.thalmann@epfl.ch
}

\begin{abstract}
By nature, human beings perceive their environment mostly using sight and audition. Vibrotactile feedback has proven satisfying results in the domains of simple multimodal interaction, for immersion and navigation purposes. The scope of this research is to evaluate the additional value of tactile feedback on the user upper body limbs to pass 3D directional information. This paper presents the development of a vibrotactile jacket and its software interface. A validation concept test bench has been setup to measure the effect of our vibrotactile device onto the response time to localize a target in a virtual environment over visual and auditory cue. Early results are encouraging by showing clear benefits of our vibrotactile system while the complexity of the multimodal environment is increasing.
\end{abstract}

\section{INTRODUCTION}

Most of complex information about our surrounding world is recovered by our vision system. We recognize colours, persons, objects, signs, writing and movements via our visual cues. The audition system plays also an important role in complex information integration by being the natural media for oral communication.

In our research we have been interested in piloting and driving security improvement. The main idea is to provide alert information without saturating already overloaded perceptual cues. Audio and visual warnings such as horns for cars, or stalling alerts in plane are commonly used as critical alerts. Audio information needs mental processing that can be altered by stress, sleepiness, or motion sickness.

Our goal is to study the use of unstimulated senses such as tactile cuing of the user skin not only to alert the user but also to pass information as a direction and amplitude. We present in this paper the development of a vibrotactile jacket designed to improve response time in localizing a target among complex environments. Moreover, we present a validation concept and early testings to estimate the added value of vibrotactile feedback in cross modality with visual and auditory cues.

At first, we will present related work using multimodal feedback and existing vibrotactile systems. Then, we will justify our choices made for designing our vibrotactile system. The description of hardware and software implementation will follow. Finally, we will describe the current test bench development and the early test results and discussions.

\section{RELATED WORK}

Research on the skin sensitivity to various tactile stimuli has been studied as another communication cue for more than half a century [1, 2]. Using the tactile cue for communication shows that information seems naturally proficient at providing low level information and suitable for reflex training.

Tests have been performed to estimate the ability to judge the locations of a vibrotactile stimulus presented at different location on the user torso [3]. Authors conclude that the threshold to distinguish two different stimuli is about $8 \mathrm{~cm}$ in the middle section of the torso. One important effect which has to be taken into account is the temporal masking [4] that induces a reduction of the skin sensitivity to a prolongated stimulus.

Vibration feedback is used for a decade in commercial devices for video games. The earliest success example is the Dual Shock controller which was first made public at the playstation expo in november 1996. It is mainly used as a limited force feedback which, for example, alerts collision in racing games. The vibration of this controller definitely increases the immersion of the user in the game and is still embedded in the last generation of controllers for playstation 3 and Nintendo Wii. The company Philips, released recently the amBX system [5]. It is mainly based on a dynamic ambient light system combined with audio surround. But it also includes some tactile feedback through a vibrating rumble pad mounted into the keyboard and two fans blowing winds at the user face. These examples show the growing interests of the gaming industry in this technology.

The improvement of immersion is a challenging topic for virtual reality research. Thus, an important number of prototypes have been developed to provide fingertip stimulation [6]. It shows encouraging results especially in reducing blindness handicap [7]. Applications of larger vibration systems, such as jackets or other clothes, have been studied and perform well on first person shooters for example [8]. It indicates to the user where danger is coming from and improves reaction time to counter attack.

Many researches are going on the use of vibrating systems to enhance navigation and environment awareness [9,10,11], especially when visual feedback is reduced, absent or already overloaded. Most of those applications aim at providing spatial information to help the user locating himself or his 
vehicle according to the surrounding environment. One example is the research done on vibrating belts for navigation way point enhancement [12]. The device consists in a belt containing 8 vibrators which vibrates according to the position of the target given by GPS coordinates. The system has been tested on board of a helicopter and a boat to detect its influences on the trajectory taken by pilot with or without tactile feedback. Prototype of tactile display onto the user body have also been tested on aircraft crew members by the Naval Aerospace Medical Research Laboratory for enhancing spatial orientation and situation awareness in military applications[13].

Joined research, done at the department of experimental psychology at the University of Oxford and the Transport Research Laboratory of Wokingham, studies the influence of vibrotactile collision warning signals in driving simulation [14]. Their setup was using two vibration actuators fixed on the user belt, one in front and one in the back. The driver also has a three state visual indicator to show if he is too close, too far or just at a good distance from the car ahead. The trial is to follow a car which will accelerate and brake at random time with disabled brake lights. This experiment shows a great improvement in response time while using the vibrotactile system over visual cues using or not the three state indicators.

This last research inspired us to take a step further and develop our own system. In the next section, we deal with our system requirements and justification.

\section{VIBRATION FEEDBACK}

In a previous research, we developed a vibrotactile system based on a vibrating belt containing 8 actuators providing direction information of obstacles for tele-operation application [15]. This experiment shows that our system was very sensitive to the contact quality between the actuators and the user skin. It was limited to 2 degrees of freedom by its own design, i.e. the vibration feedback was constrained in a plane around the user's waist so it can only provide directional information in the horizontal plane.

This development aims at extending our concept and bringing it to a new level of usability. Our first concern is to develop a device that allows the user to render $3 \mathrm{D}$ information via vibrotactile feedback. The main constraint is that the system has to be perfectly wearable, just as a second skin, so the user can interact freely with the surrounding environment. It also has to be reconfigurable to be adapted to a large panel of different applications.

In order to be able to give information about 3D directional information, we have to create a continuous vibrotactile stimulus around the user in 3D space. We choose to cover a large surface of the user body. For practical purposes we concentrate on the upper body torso and limbs, and embed the whole system into a light sport jacket.

One of our concerns is also to provide a continuous feedback in space and not singular stimuli points. As shown in the related work, the skin sensitivity of the upper body limbs is around several centimetres. So we decided to implement enough actuators to match the resolution of our natural cues.

Since the system would be worn by different people on the top of different garment, we ensure by multiplying the actuators that the effect of having variation on contact quality with the user skin will be counterbalanced by having a redundancy of actuators in the jacket.

We use 32 actuators to cover the torso and the arms of the user. They are placed as shown in the Fig 1 . The intensity of the stimuli is computed by taking the dot product between the relative normalized position of the actuator and the given direction. A sensitivity curve allows for tuning the correlation between the desired feedback and the given input parameter.

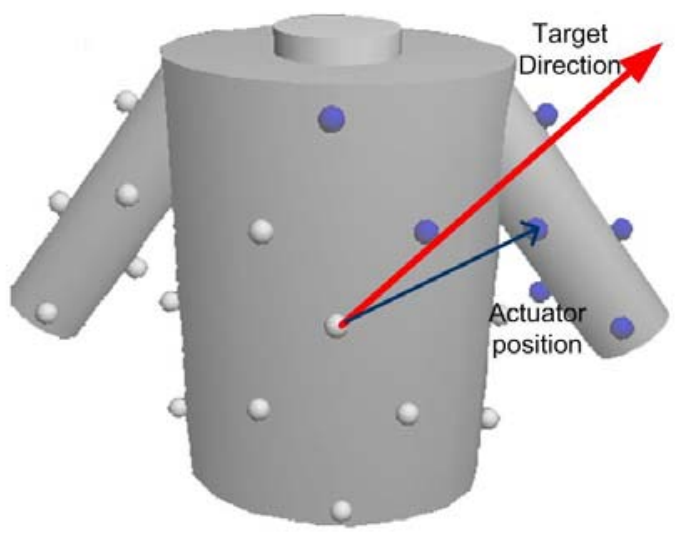

Fig 1 Vibration feedback

The system will implement different modes of vibration feedback in order to compare and determine which one renders the directional information in the most accurate way. Three modes of vibrations caught our attention: continuous, alternative and wave mode. The continuous mode provides a vibration stimulus directly function of the input direction and amplitude. The alternative mode answers to the problematic of the attenuation of skin sensitivity due to overwhelming of the biological skin sensors by producing burst of vibration with different amplitudes. The wave mode produces series of waves of vibration which displace along the given direction. In this mode, the amplitude of the input is translated into different speed for the waves.

The next part will describe the hardware and software system implementation and related technical information.

\section{VIBROTACTILE DEVICE DEVELOPEMENT}

The Fig. 2 shows a picture of the actual system in use. It is composed of a vibration jacket, an embedded system and a computer. The jacket contains the actuators driven by the embedded microcontroller. The computer runs the user interface, computes the desired vibrating feedback and sends the command to the microcontroller board. 


\section{A. Vibration Jacket}

The actuators are mounted onto a standard sportswear jacket, light and comfortable. We used flat DC vibration motors issued from mobile phone technology placed to stimulate the user upper body skin. Those actuators are powered with a PWM signal of 5 Volts of amplitude to modulate the energy transmitted. The overall electrical consumption is around $100 \mathrm{~mW}$ per actuator at maximum level intensity. In order to improve comfort of the user, we used electrical wires embedded in soft tissue naps. The system is powered by a $7.4 \mathrm{~V}$ and $800 \mathrm{~mA} / \mathrm{h}$ small Lithium Polymer battery like the one used to power RC helicopters.

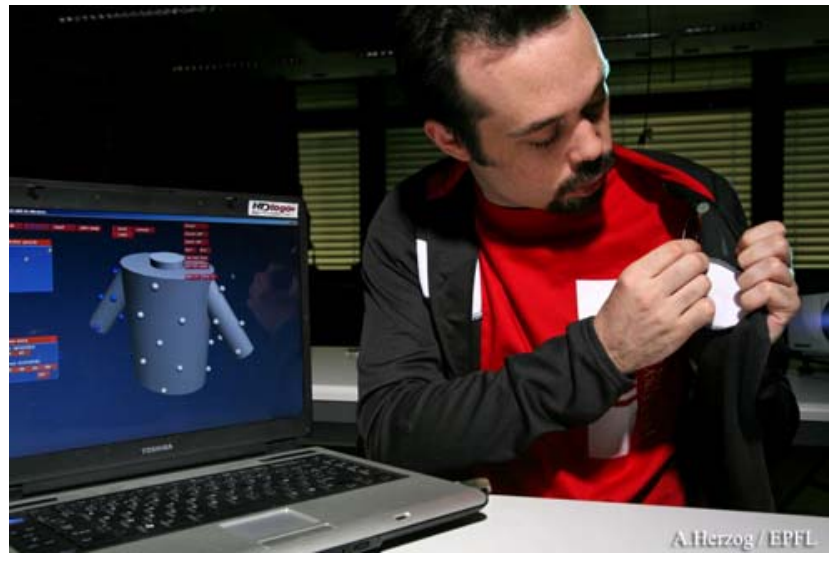

Fig. 2. Vibrotactile vest and configuration interface.

\section{B. Embedded System}

The embedded system is based around a home made microcontroller controller board. The information treatment is done by an 8bits architecture PIC18 microcontroller from Microchip Company. This chip contains a small processing unit running at $40 \mathrm{MHz}$ with various input and output such as PWM (Pulse Wide Modulation) controllers, UART manager, analogue to digital converters, and internal memory of $64 \mathrm{kB}$. The device is programmed in assembly code and handles the communication to the computer as well as the command of the 32 Darlington transistors which drives each vibration motor.

Since the microcontroller does not contain enough PWM controllers, the 32 outputs are software emulated using standard input/output pins. We used a memory buffer, the PWM stack, which contains the different states of the system during a PWM period and use counter based interrupt approach to pass from one state to another. The PWM period is fixed to $10 \mathrm{~ms}$ to not overload the processing unit and not disturb the communication treatment function.

The communication is done via serial communication through the UART port of the microcontroller. A Bluetooth module is connected to provide serial communication. The data are embedded in small packet containing header type, size, data and a checksum to insure reliable data transmission. The different types of transmitted packages are "start”, "stop", "status interrogation" commands, and an "update PWM stack" data packet.

\section{Calibration and integration interface}

A computer is used to command the vibrotactile jacket. It gathers information from the external environment and computes the desired feedback output.

The interface, as shown on the laptop in Fig.2, presents a schematic representation of the vest with its actuators. The user can add, remove and displace virtual actuators in 3D space to match position of the real ones. Each actuator can also be assigned to the desired physical output. As all the settings information, actuators configuration can be saved and reloaded according to the application and the hardware. The user can also setup overall sensitivity of the power applied to the vibration motor over the related input. The sensitivity of each vibration motor can be singularly modified to allow compensating the perception difference due to variation of skin sensitivity or contact to the skin.

Each actuator's value is computed at each time step and stored into the PWM stack which is sent to the microcontroller via one of the serial COM port of the computer. External information to the system parameters is gathered through an UDP server implemented in the interface system. It can take custom parameters to compute a direction and the amplitude of the vibration to render. The system implements the three different vibration modes. Continuous vibration represents an output value directly function of the input value. Alternative vibration produces burst of vibrations of different intensity. The last mode creates "waves" of vibrations which displaced in time along the given direction.

The next part presents our test bench application to estimate the added value of the different mode of our vibrotactile system over visual and auditory cues.

\section{TEST BENCH}

Our goal is to validate the use of vibrotactile feedback to provide accurate and intuitive direction in space. We propose to immerse the user into a controlled virtual environment which presents abstract scenery composed by different visual objects seen through a Head Mounted Display (HMD), audio environment rendered with a 5.1 surround speaker system, and vibrotactile feedback via our vest. The task will be to find a randomly placed object in the scene using combination of multimodal information. We will measure the response time to localize and validate the visual target with the different vibration modes to estimate their added values.

\section{A. System description:}

The test bench is composed by our vibrotactile system with its configuration interface, a graphic workstation which runs the virtual environment connected to a HMD, a 5.1 surround system and an inertial tracker to acquire the user head orientation as shown in Fig.3.

To render the virtual scenery to the user, we use a HMD Kaiser XL50. It contains two miniatures LCD screen which 
can display two different videos, one for each eye, with a resolution of $1024 * 768$ pixels at $60 \mathrm{~Hz}$. Thus, it can provide stereoscopic vision to naturally pass depth information. An inertial sensor, the InertiaCube3 is used to track the orientation of the user head. This tracker is very accurate and has a refresh rate of $180 \mathrm{~Hz}$ and a latency of $2 \mathrm{~ms}$ due to the integration of the accelerometers. It provides three angles value for yaw, pitch and roll with a range of 360 degrees on all axes and is thus capable of tracking the head's movements. This is used to map the movement of the user to the virtual camera in the rendered scene.

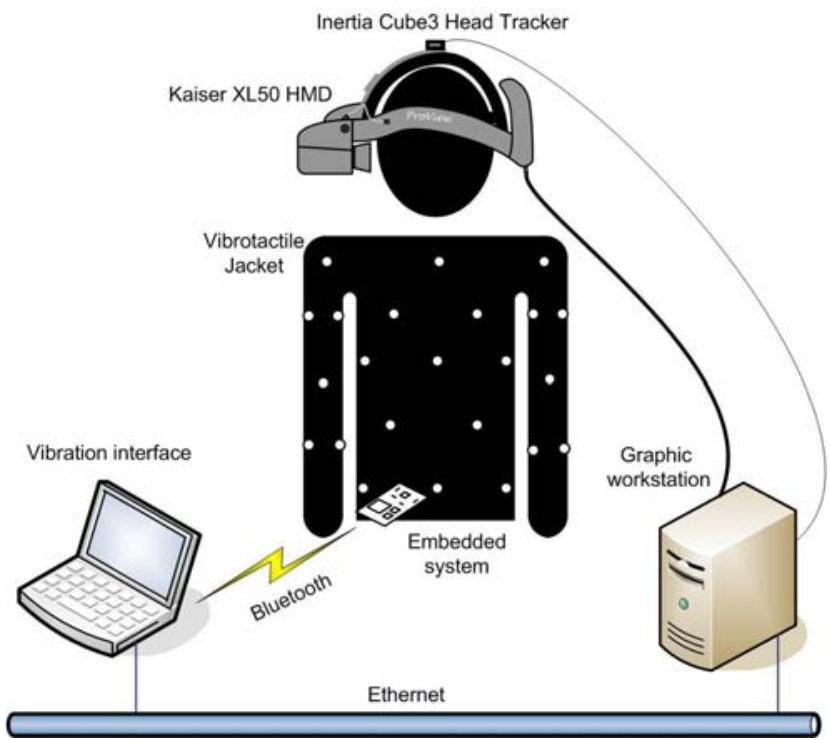

Fig.3 Overview of the test bench

The sound environment is rendered through the embedded personal computer running the virtual environment. 5.1 speaker packs is linked to the outputs and surround the user with appropriate sound. By using the $\mathrm{C}++$ library FMod, we create different sound sources localized in space which plays respecting wave files.

A personal computer renders the virtual environment. The library MVisio [16], developed in our laboratory, implements a user friendly graphic engine based on OpenGL. It contains high level functionalities handling such as scene graph management, lightning, 2D GUI, camera, object importation and other rendering control. A UDP client is used to handle communication via Ethernet with the computer controlling the vibration feedback system. It sends every $20 \mathrm{~ms}$ the direction and distance of the given target in the virtual environment.

\section{B. Test scenario}

In order to evaluate the added value of our system to pass direction information in a multimodal environment, we define the following test scenario.

During the trials, the user is standing in the void with no substantial referential about its own positioning. He/she is able to look freely around in all direction including above and under him/her. Spheres of different sizes, colours and textures are moving and spinning. The user goal in this experiment is to localize the single ball presenting a texture of a white rabbit drawing. Each sphere texture is associated with a particular sound pattern played using spatial sound rendering taking in account the positioning of the object as well as its size to determine position and volume of the resulting sound. The vibration feedback is transmitted through the actuators of the vest using one of the three vibration models, continuous, alternative or waves they only respond to the target direction.

An instruction interface is modifying visual, audio and vibrotactile feedback by changing the parameters in real time from trials to trials. While the objective is reached the simulation resets and reinitialises using a new set of predefined parameters. The number, size, displacement and rotating speed of the spheres changes and alter the difficulty to visually find the target. For sound feedback we modify the ratio between the radius of the sphere and the sound volume associated. The vibration can be switched from one mode to another or even disabled. The most important aspect to study is the pertinence of each way of communication by modifying the contribution of the target over all the other elements. All the parameter can be singularly randomized using uniform distribution for studying the influence of each parameter among the others. It is also possible to inverse logical use of the different parameters to evaluate confusion introduced by contradiction stimulation, for example making the small spheres louder than the big ones.

The user will go through a large series of trials. Each trial will measure the time for the user to find the target. To do so, he/she fixes his/her head in the direction of the target and hold still for a full second to indicate validation. This second will be subtracted to the final response time value to not alter the interpretation of the results. Before running the measurement, we will start by presenting separately the visual target and it associated sounds. A short explanation will be given also about the different possible feedback and parameter range values. After 20 blank trials to insure that the user understands and get used to the system we start recording response time values. The following part will comment the first results from those experiments.

\section{DISCUSSION AND FUTURE WORK}

Early results provide us enough information to describe general behaviour and user acceptance to the use of multimodal cues for direction determination.

The perceived accuracy of vibrotactile or audio feedback by themselves is too low for an exact localisation of the target without visual target confirmation. Although we observed major reduction of the search time with small targets or when surround by a large amount of other elements. The user is immediately informed of the approximate position of the target and aims quickly in the proper area of the scenery. The time to acquire the visual target is then greatly improved.

Vibrotactile cue seems slightly more efficient than sound feedback in our setup. The user concentrates more 
thoughtfully to analyse where the sound is coming from. Although one great advantage of the auditory system is that even with noise coming from the other spheres, the user is able to distinguish and intuitively isolate the sound of the target. This effect is almost impossible with a vibration system which has to provide one unique simple type of information to be understandable. Among the different vibration mode the wave mode doesn't seem to perform properly and is found confusing by some of the testers. Continuous vibration mode shows encouraging results on the response time, but the alternative vibration mode seems a bit better in term of performances and user acceptance. This is mostly due to the skin overwhelming of a continuous vibration excitation which does occur while summated to burst of vibration stimuli. As general trend, we observe a clear improvement of response time due to the use of our vibrotactile feedback while the visual information is overloaded.

A large number of trials still need to be performed to determine precise influence of relevant parameters. We are currently working at making a second vibrotactile vest to fit larger people to run tests on a more substantial population. We want to distinguish the influence of the different parameters and cues of the simulation over the response time. Interesting improvement to the actual setup will be to add sound coming from under and above the user to avoid bias in the influence of sound feedback due to the vertical component of the direction of the target. Another point would be to capture the posture of the user upper body and integrate it in the vibration feedback system to insure cohesion of the produced vibration in space with the user proprioceptive cues. Actually our system just considers the user standing with his arm along its sides which doesn't match always match the reality.

\section{CONCLUSION}

The development of our vibrotactile system shows clear improvement on perceiving directional information in cross modality with visual and audio feedback.

Application of such principles is highly valuable in driving and piloting ground or aerial vehicles for alert purposes. During stressful situations, response time is critical. The time to analyse visual indicators or understand audio commands can lead to dramatic issues. We are actually studying the interest of such a system to follow predefined trajectory during takeoff and landing approach for regular flight and also in handling exceptional plane behaviour.

\section{ACKNOWLEDGMENT}

We would like to thank the Intuition European network of excellence for sponsoring this research, our colleague Achille Peternier for the development of the MVisio Library during his PhD, Alain Herzog photograph at EPFL and Nicolas Fortini for his work on the vibration interface during his master thesis.

\section{REFERENCES}

[1] Gilmer, B.v.H.,1960. Possibilities of cutaneous electro-pulse communication. In: Hawkes, G.R. (Ed.), Symposium on Cutaneous Sensitivity. U.S. Army Medical Research Laboratory, Fort Knox, KY, pp. 76-84.

[2] Hennessy, J.R., 1966. Cutaneous sensitivity communications. Hum. Factors 8, 463-469.

[3] Erp, J.B.F. van \& Werkhoven, P.J. (1999). Spatial characteristics of vibro-tactile perception on the torso.TNO-Report TM-99-B007. Soesterberg, The Netherlands: TNO Human Factors.

[4] J.C. Craig, Temporal integration of vibrotactile patterns, Percept. Psychophys. 32 (1982), pp. 219-229.

[5] (2008) The amBX website [online] available http://ambx.com

[6] Malik, S. and Laszlo, J. 2004. Visual touchpad: a two-handed gestural input device. In Proceedings of the 6th international Conference on Multimodal interfaces (State College, PA, USA, October 13 - 15, 2004). ICMI '04. ACM, New York, NY, 289-296.

[7] Kamel, H. M. and Landay, J. A. 2000. A study of blind drawing practice: creating graphical information without the visual channel. In Proceedings of the Fourth international ACM Conference on Assistive Technologies (Arlington, Virginia, United States, November 13 - 15, 2000)

[8] Lindeman, R. W., Page, R., Yanagida, Y., and Sibert, J. L. 2004. Towards full-body haptic feedback: the design and deployment of a spatialized vibrotactile feedback system. In Proceedings of the ACM Symposium on Virtual Reality Software and Technology (Hong Kong, November 10 - 12, 2004). VRST '04. ACM, New York, NY, 146-149.

[9] Ho, C., Tan, H.Z., Spence, C., 2005. Using spatial vibrotactile cues to direct visual attention in driving scenes. Transport. Res. F: Traffic Psychol. Behav. 8, 397-412.

[10] Burnett, G.E., Porter, J.M., 2001. Ubiquitous computing within cars: designing controls for non-visual use. Int. J. Hum-Comput. Stud. 55, 521-531.

[11] Rochlis, J.L., Newman, D.J., 2000. A tactile display for International Space Station (ISS) extravehicular activity (EVA). Aviat. Space Environ. Med.71, 571-578.

[12] J. Van Erp, H. Van Veen, C. Janseen, T. dobbins, (2005) Vibrotactile Waypoint Navigation at Sea and in the Air: two Case study, ACM Transactions on Applied Perception,

[13] Rupert A.H:, McGrath, B.J., Griffin M.(2002).A Tool to Maintain Spatial Orientation and Situation Awareness for Operators of Manned and Unmanned Aerial Vehicles and other Military Motion platforms, RTO HFM Symposium on "spatial Disorientation in Military Vehicles: Causes, Consequences and Cures”, 31.1-31.15.

[14] Cristy Ho, Nick Reed, Charles Spence, Assessing the effectiveness of 'intuitive' vibrotactile warning signals in preventing front-to-rear-end collisions in a driving simulator, Accident Analysis \& PreventionVolume 38, Issue 5, , September 2006, Pages 988-996.

[15] X. Righetti, S. Cardin, D. Thalmann and F. Vexo, Immersive flight for surveillance applications, IEEE Symposium on 3D User Interfaces, pp. 139-142, 2007.

[16] A. Peternier, D. Thalmann and F. Vexo, Mental vision: a computer graphics teaching platform, Proc. Edutainment in Lectures Notes in Computer Science, pp. 223-232, 2006. 